\title{
A Survey of PHD Filtering Method Based on Random Finite Set
}

\author{
Yan Song ${ }^{\mathrm{a}}$,Jianwang $\mathrm{Hu}{ }^{\mathrm{b}}$ and Bing $\mathrm{Ji}^{\mathrm{c}}$ \\ School of Ordnance Engineering College, Shijiazhuang, 050003, China. \\ a1849278046@qq.com, bm18104441502@163.com, cm15610812761@163.com
}

Keywords: random finite set, multi-target tracking, probability hypothesis density filter

\begin{abstract}
Probability hypothesis density filter based on Random Finite Set(RFS)recently become a research hotspot for multi-sensor information fusion in the world. An overview of the emergence, the development and the present research situation of the PHD filter in target tracking is presented here. Special attention is paid to the following areas:multi-target tracking method, method of PHD filter based on random finite set and the research status of probability hypothesis density filter. Finally, the future research directions in probability hypothesis density.
\end{abstract}

\section{Introduction}

As one aspect of the development of science and technology, multi-target tracking [1]can be traced back to the eve of the second world war, and the basic concept was first proposed by War in the mid 50s. In 1960, Kalman proposed the Calman filtering algorithm [2], and was successfully applied to the field of target tracking. Since then, the research of multi-target tracking method has been paid more attention. the scientists of Bar-Shalom, Singer, Blair, Reid, Blom, Blackman and Chong as the representative have made great contributions in maneuvering target tracking and data association, but also put forward a number of classic algorithms. In the last few years, the number of targets is changing with time, and the number of targets is unknown. In this case, the multi-target tracking problem becomes complicated and difficult. Thus, Martin and Mahler proposed the probability hypothesis density filter (Probability hypothesis density, PHD) tracking algorithm based on the random finite set (Random Finite Set) theory. This method breaks through the traditional data association [3] method and avoids the problem of data association. In the complex environment, this method can solve the problem of multi-target tracking and become a hot topic in the field of multi-target tracking. The technology has important theoretical value and broad application space in the fields of military, aviation and civil.

\section{Traditional Multi-target Tracking Algorithm Based on Data Association}

In the previous research, the main problem to be solved in the multi object tracking problem is the correlation between the observed value and the target. Traditional tracking algorithm has Nearest Neighbor Data Association [4, 5] (NNDA), Probabilistic Data Association [6, 7] (PDA), Joint Probabilistic Data Association [8] (JPDA) and Multiple Hypothesis Multi Hypothesis Tracker Tracking [9](MHT). In the above method, the problem of multi-target tracking is transformed into a single target tracking problem, which is the core and key of data processing. The simplest data association algorithm is the nearest neighbor method, which has the advantages of less computation and easy implementation; The Probabilistic data saaociation(PDA) algorithm is the measurement data of all the weighted average of the tracking threshold in the sense of probability, and as the filter output to obtain more posterior information based data association. The advantage of the algorithm is that the probability of losing the target and the following error is small and the computational cost is relatively small. However, the algorithm can only be used for single target tracking in clutter environment, and the phenomenon of error following or filtering divergence occurs when the target is relatively concentrated or the clutter density is large. In order to deal with the multi objective situation, Bar-Shalom proposed the Joint Probabilistic Data association (JPDA). The algorithm is applied to the 
target number of known, and the algorithm is easy to cause the target track "aggregation" in dealing with dense targets and a similar distance or track crossing situation, to result in leakage, or with error, to target track merging problem in target tracking, and to measure the number of goals or more. There will be a group of related explosion "problem increasing the computational burden. In 1978, Reid proposed a multiple hypothesis tracking (MHT) algorithm based on the global optimal algorithm. This algorithm uses the validity matrix to generate a number of assumptions, then calculate the probability of each hypothesis and delete the assumption of low probability, finally form a measurement point and track matching. The algorithm is optimal in theory, but in fact we need to know some prior target and value of detection system, such as the number of targets, the false alarm density, target detection rate, which is also obtained under normal conditions. In addition, the algorithm has the defects of large computation, and is not conducive to real-time processing.

The common thing about the above method is that the tracking process needs to be associated first. Relevance is the core and key to tracking the problem. If the association is wrong, the estimate will be poor. In this case, the algorithm requires a high degree of accuracy of the association, so the computation is very large. And when the number of targets is high and there is a high degree of clutter and missed rate, the association itself is difficult to deal with. And there will be problems such as combined explosions, exponential growth and so on. In fact, when the number of targets is large, the data association problem is more complex than the tracking problem itself, and the problem has become a bottleneck in the application of multi-target tracking algorithm.

\section{Multi-target Tracking Based on Random Finite Set}

At present, in the field of multi-target tracking, because the random finite set (RFS) multi-target tracking does not need data association, the calculation of the algorithm is greatly reduced, which has aroused great attention from domestic and foreign scholars.

In Random Finite Set, the target state and the measurement can be expressed as:

$$
\begin{gathered}
X_{k}=\left\{x_{k, 1}, x_{k, 2}, \cdots, x_{k, N_{k}}\right\} \in F(\chi) \\
Z_{k}=\left\{z_{k, 1}, z_{k, 2}, \cdots, z_{k, M_{k}}\right\} \in F(\psi)
\end{gathered}
$$

PHD filter is mainly through the PHD prediction and update to achieve the recursion of the algorithm in order to complete the Multi-target Tracking.

The predicted PHD can be expressed as:

$$
\begin{aligned}
& D_{k \mid k-1}\left(x_{k} \mid Z_{1: k-1}\right)=\gamma_{k}\left(x_{k}\right)+\int D_{k \mid k-1}\left(x_{k} \mid x_{k-1}\right) D_{k-1}\left(x_{k-1} \mid Z_{1: k-1}\right) d x_{k-1} \\
& \quad \text { with } \\
& D_{k \mid k-1}\left(x_{k} \mid x_{k-1}\right)=b_{k \mid k-1}\left(x_{k} \mid x_{k-1}\right)+P_{s} f\left(x_{k} \mid x_{k-1}\right)
\end{aligned}
$$

where $\gamma_{k}\left(x_{k}\right)$ is the new target of PHD.is derived $\mathrm{t} b_{k \mid k-1}$ arget of PHD; $P_{s}$ is survival probability; and $f\left(x_{k} \mid x_{k-1}\right)$ is State transition probability density.

When the measurement of time $\mathrm{k}$ is effective, its update PHD can be expressed as:

$$
\begin{aligned}
& D_{k}\left(x_{k} \mid z_{1: k}\right)=\left(1-P_{D}\right) D_{k \mid k-1}\left(x_{k} \mid z_{1: k-1}\right) \\
& +\sum_{z \in Z_{k}} \frac{P_{D} D_{k}(z)}{\lambda_{c} c_{k}(z)+P_{D} D_{k}(z)} D_{k}\left(x_{k} \mid z_{k}\right) \\
& \quad \text { with } \\
& D_{k}(z)=\int g_{k}\left(z \mid x_{k}\right) D_{k \mid k-1}\left(x_{k} \mid Z_{1: k-1}\right) d x_{k}
\end{aligned}
$$

and

$$
D_{k}\left(x_{k} \mid z_{k}\right)=\frac{g_{k}\left(z \mid x_{k}\right) D_{k \mid k-1}\left(x_{k} \mid Z_{1: k-1}\right)}{D_{k}(z)}
$$

where $P_{D}$ is detection probability; $g_{k}\left(z \mid x_{k}\right)$ is likelihood function; $\lambda_{c}$ represents the average number of clutter per scan (usually assumed to be a Poisson distribution) and $c_{k}(z)$ is Clutter distribution function. 


\section{Research status of probability hypothesis filter}

Although the Probability hypothesis density (PHD) filter replaces the target tracking of the traditional data association method and overcomes the series of problems caused by data association, the PHD framework itself has its own problems, such as large computational complexity and precision low degree of difficulty.

(1) The problem of low precision and large calculation

Erdinc [10] said that if the number of targets can be added to the two order PHD filter which will be improve PHD filter performance. Based on the above recommendations, the CPHD [11, 12] (Cardinalized PHD, CPHD) filter is proposed. The filter is used to predict and update the target PHD and the potential distribution, which makes the estimation of target number more accurate. In the clutter environment, the probability hypothesis density (PHD) is prone to the loss of distribution information, which leads to the deviation of target detection. A multi-target tracking algorithm combining the potential probability hypothesis density (CPHD) filtering and the smoothing algorithm is proposed in literature [13]. The algorithm makes up the shortcomings of PHD filter to lose high order potential distribution information, and further improves the estimation performance of multi target state.In the literature [14], the performance of the three algorithms of PHD, CPHD and JPDA is compared, and the calculation cost of JIPDA is the highest, but the accuracy of target tracking is the most accurate. CPHD is the new goal Low response time. In literature [15], the irrationality of the iterative updating method is analyzed, that is, the difference of the sensor iteration order will lead to the inconsistency of the update result. In this paper, the PHD update formula is expressed as the product of the PHD pseudo-likelihood of the sensor, and the multi-sensor PHD (PMPHD) and the product multisensory cardinalized PHD (PMCPHD) filter are proposed by relaxing the hypothesis. The analysis shows that PMCPHD has a potential advantage in computing; In the literature [16], the SMCPHD algorithm is used to obtain the local state estimation value for each sensor. Then, the multi-sensor multi-target state fusion result is obtained by the sequential fusion method. When the target model and the measurement model are nonlinear, the multi- PHD tracking accuracy of the target is very high. Mahler uses the simplified clutter model to propose LC-CPHD (Linear-complexity CPHD) [17] filter, which improves the computational performance, reduces the computational complexity and improves the target tracking accuracy. In literature [18, 19], SMC and GM techniques are proposed for approximate solution. The SMC method uses a set of particles with weights to approximate the multi-objective PHD, and the Bayesian filter is used to recursively track the particles and their weights to achieve multi-objective tracking. The GM method uses a set of Gaussian component weighted sums to approximate the multi-objective PHD. By recursive Gaussian component mean, weight and covariance matrix to achieve the multi-objective PHD recursive update, and then achieve multi-target tracking The Both methods reduce the computational complexity and improve the accuracy of target tracking. In order to further improve the efficiency of GMPHD, the literature [20] introduces the pruning method and the method of combining the same Gaussian term to reduce the computational complexity. In the literature [21], Daniel compares the advantages and disadvantages of the two algorithms SMCPHD and GMPHD, and then proposes the Gaussian particle rules (GMPPHD) of PHD filter, and still uses the mixed Gaussian term to approximate PHD, Since the filter does not exist in the specific form of analysis, so the Monte Carlo integration method to solve the PHD recursive formula.

(2) the problem of difficult to track

In literature [22], a PHD trajectory maintenance algorithm based on fuzzy clustering is proposed. The algorithm makes full use of multi - frame information, carries on the multi - step prediction to the current time state, and weights according to the inertia, and then uses the fuzzy clustering to obtain the membership degree of the current estimation belongs to each track, finally obtains the track.In the literature [23], the Gaussian hybrid PHD (GMPHD) is used to filter the measurement information of the multi-sensor, and then the nearest neighbor data is processed by the filtering result, and the multi-target track is obtained. The method can effectively improve the filtering accuracy, reduce the 
amount of computation, and improve the accuracy of data association. In literature [24], an important sampling function design method based on rms Kalman filter (SCKF) and statistical threshold technique is proposed. The algorithm has the advantages of high precision and stable estimation result in nonlinear multi - target tracking.

(3) The problem of non-linearization

For the nonlinear system, in literature [25], two implementations are given: Extended KalmanProbability Hypothesis Density (EK-PHD) filtering and no-trace Kalman-Probability Hypothesis Density (UK-PHD) filtering method are proposed. The Extended Kalman-Probability hypothesis density filtering method is a non-linear system function for the first-order Taylor formula. Although the method is less complex, but the accuracy is not high; The non-trajectory Kalman-probability hypothesis density filtering method is to select the weighted sampling point to approximate the probability distribution of the nonlinear function, and then obtain the recursive relation of the multi-objective probability hypothesis density to realize multi-target tracking. Compared with the extended Kalman - probability hypothesis density filtering method, this method has higher filtering accuracy. In 2010, Macagnano [26] proposed another nonlinear Gaussian system of probability hypothesis density filtering method, that is, volume Kalman-Probability Hypothesis Density (CK-PHD) filtering method. This method combines the Cubic Kalman Filter (CKF) method with the probability hypothesis density filtering algorithm to obtain the recursive form of the probability hypothesis density. The method has the advantages of small computational complexity and stable performance of the filter.

\section{Prospects for Future Research}

(1) PHD implementation method:

Although the majority of scientific research workers now use GM and SMC and other algorithms, these algorithms have the great limitations. For example, although the GM class algorithm has a high precision for the target tracking under the linear Gaussian hypothesis, the tracking accuracy is greatly impaired under non-linear conditions. SMC algorithm is difficult to choose the appropriate importance of density function, and there are a lot of computing problems. These issues need to be studied in depth.

(2) PHD track analysis:

As the PHD filtering method itself can not directly output the target state and track information, therefore, in the future study, we should focus on how to extract the peak extraction technology and track extraction technology into the PHD filter frame structure, so that the Track for accurate extraction.

(3) PHD filter performance study:

It is inaccurate to determine the performance of the PHD algorithm only by distance error and potential error. Should be based on realistic assumptions on the basis of the reduction, through a large number of simulation experiments, a variety of data or image comparison, to draw the final conclusion.

\section{Summary}

Because the PHD filtering method avoids the traditional data association, it is very important. After recent years, the research on multi-target tracking method based on PHD framework is developing in an increasingly precise direction. As a new theoretical architecture, will promote the development and application of information fusion theory.

\section{References}

[1]N.Wax.Signal-to-noke improvement and the statistics of tracking populations[J]. Journal of Applied Physics, 1995,26(5):586-595. 
[2]Kalman R E. A new approach to linear filtering and prediction problems. Journal of Basic Engineering, 1960,82(1):35-45

[3] Han Chongzhao, Zhu Hongyan, Duan Zhansheng and so on. Multi - source information fusion [M]. Beijing: Tsinghua University Press, 2006

[4]Singer R A, Sea R G. New results in optimizing surveillance system tracking and data correlation performance in dense multitarget environments[J]. Automatic Control IEEE Transactions on, 1973, 18(6):571-582.

[5] Tian Hongwei, Jing Zhongliang, Hu Shiqiang et al. Multi-frame nearest neighbor data association algorithm based on multi-rate motion model [J]. Journal of Shanghai Jiaotong University, 2005, 39: 413-416.[6] Y. Bar-Shalom, E. Tse. Tracking in a cluttered environment with probabilistic data association[J].Automatica, 1975,11(5):451-460

[7] Li Weitong. Probability data association algorithm in dense environment [J]. Journal of Projectiles, Rockets and Guidance, 2004: 362-363.[8] T. E. Fortmann, Y. Bar-Shalom, M. Scheffe. Sonar tracking of multiple targets using joint probabilistic data association[J]. Oceanic Engineering IEEE Journal of, 1983, 8(3):173 - 184.

[9]Muthumanikandan P, Vasuhi S, Vaidehi V.Multiple Maneuvering Target Tracking Using MHT and Nonlinear Non-Gaussian Kalman Filter[C] Signal Processing,CommunicaTions and Networking, 2008.International Conference on IEEE,2008:52-56.

[10]Erdinc O, Willett P, Bar-Shalom Y. Probability hypoth-esis density filter for multitarget multisensor tracking. In:ProceedinGs of the 8th International Conference on Infor-mation Fusion. Philadelphia, PA: IEEE, 2005. 25-29

[11] Mahler R. A theory of PHD filters of higher order in target number.In: Proceedings of the 2006 Signal and DataProcessing of Small Targets.Orlando,FL:SPIE,2006.6235K-62350K-1210.

[12]Mahler R. PHD filters of higher order in target number. IEEE Transactions on Aerospace and Electronic Systems,2007, 43(4): 1523-1543

[13] XIE Xing-xiang, CAI Ruo-hua, WU Sun-yong et al.Multi-target tracking algorithm based on density probability hypothesis density smoother [J]. Journal of Guilin University of Electronic Technology, 2015,35 (2) 121-126.

[14]M. Beard, S. Arulampalam. Performance of PHD and CPHD filtering versus JIPDA for bearings-only multi-target tracking[C]. Information Fusion (FUSION), 2012 15th International Conference on. IEEE, 2012:542 - 549.

[15]Mahler R. Approximate multisensor CPHD and PHD filters[C].Proceedings of the 13th International Conference on Information Fusion. Edinburgh, UK: IEEE, 2010. 1-8

[16]Meng F B, Hao Y L, Xia Q X, Ouyang T S, Zou W. A particle PHD filter for multi-sensor multi-target tracking based on sequential fusion[C].Proceedings of the 2009 International Conference on Information Engineering and Computer Science. Wuhan, China: IEEE, 2009. 1-5

[17]Mahler R. Linear-complexity CPHD filters[C].Proceedingsof the 13th International Conference on Information Fusion. E-dinburgh, UK: IEEE, 2010. 1-8

[18]Vo B, Singh S, Doucet A. Sequential Monte Carlo methods for multitarget filtering with random finite sets[J].IEEE Transactions on Aerospace \& Electronic Systems, 2005,41(4:) 1224-1245.

[19] Vo B, Pasha A, Tuan H D. A Gaussian Mixture PHD Filter for Nonlinear Jump Markov Models[C]. Proceedings of the 45th IEEE Conference on Decision and Control,2006:3162-3167. 
[20]Vo B N, Ma W K. The Gaussian mixture probability hypothesis density filter[J]. IEEE Transactions on Signal Processing, 2006, 54(11): 4091-4104

[21]Clark D, Vo B T, Vo B N. Gaussian particle implementtations of probability hypothesis density filters. In: Pro-ceedings of the 2007 IEEE Aerospace Conference. Big Sky,MT: IEEE, 2007. $1-11$

[22] Ouyang Cheng, Ji Hongbing, field. A PHD path maintenance algorithm based on fuzzy clustering [J] .Acta Electronic Journal .2012,40 (6): 1284-1288

[23] Jijia, Huang Gaoming, Wu Xinhui, Ma Jie.A PHD multi-target multi-sensor correlation algorithm based on random set [J]. Electronic Information Countermeasure .2014,29 (2): 17-21

[24] Liu Zhe, Wang Zulin, Xu Ma and other SMC-PHD algorithm based on root mean square particle [J]. Journal of Beijing University of Aeronautics and Astronautics, 2015,41 (10) 1590-1598.[25]Vo B N, Ma W K. The Gaussian mixture probability hypothesis density filter[J]. IEEE Transactions on Signal Processing, 2006, 54(11): 4091-4104

[26] Mcagnano D, De Abreu G T F. Multitarget tracking with the cubature Kalman probability hypothesis density filter. The Forty Fourth Asilomar Conference on Signals,Systems and Computers [C]. Pacific Grove,USA:IEEE,2010:1455-1459 